\title{
Sleep related breathing disorders and indications for polysomnography in preterm infants
}

\section{A R T I C L E I N F O}

\section{Keywords:}

Central sleep apnea

Periodic breathing

Obstructive sleep apnea

Polysomnography

Bronchopulmonary dysplasia

Apparent life threatening event

\begin{abstract}
A B S T R A C T
There is a range of breathing problems which occur and may persist in preterm infants, such as central apneas, obstructive apneas and periodic breathing. Preterm infants may also suffer from respiratory distress syndrome and chronic lung disease necessitating prolonged use of oxygen therapy after discharge from the hospital. Due to these persistent breathing pattern abnormalities in preterm infants, there is a higher risk of altered sleep and apparent life threatening events. Polysomnography can be a helpful tool to identify those infants who have abnormalities in their breathing pattern, to identify those infants who have an increased risk to get a sleep related breathing event at home and to decide about the discontinuation of oxygen therapy.
\end{abstract}

\section{Introduction}

Sleep is very important for the optimal development of children, but is also a state in which breathing difficulties are most prominent. Preterm infants have a known risk of an immature breathing pattern or apnea of prematurity which may lead to bradycardias and desaturations both awake and while asleep. Invasive or non-invasive respiratory support or pharmacologic treatment with methylxanthines, such as caffeine or with doxapram is necessary to overcome these problems in many preterm infants [1-4]. Compared with term infants, prematurely born infants have a blunted response to a hypercapnic challenge, a diminished glossopharyngeal muscular contraction in response to periodic breathing, and a higher percentage of events due to poor sucking and swallowing that result in apnea when attempting to oral feed [5]. Furthermore, infants with persistent apnea or bradycardia may have delayed maturation of these physiologic skills and there is also a close relation between coordination of sucking and cardiorespiratory control $[5,6]$. Breathing problems may also occur due to a lack of tone in the upper airway, followed by collapse and obstruction. Many preterm infants will suffer from infant respiratory distress syndrome (IRDS), necessitating respiratory support or treatment with surfactant [7].

Most of the preterm infants will develop a mature breathing pattern and recover from the initial lung disease. However, in a number of preterm infants breathing problems can persist and can be related to inadequate maturation of the breathing pattern, obstructive breathing or the development of a chronic lung disease [8-10]. Furthermore, preterm infants are at increased risk for an apparent life-threatening event (ALTE) or brief resolved unexplained event (BRUE) [11,12]. Most of these breathing problems occur during sleep. The presence of breathing problems or unexplained events, usually leads to a stay in the hospital setting until the child has normal control of breathing during sleep. Polysomnography is a helpful tool to analyze these breathing problems. In this review we will describe the indications for the different clinical entities to perform sleep studies (polysomnography) and the consequences for further treatment and follow-up.

\section{Polysomnography}

\subsection{General}

Polysomnography (PSG) is a versatile diagnostic tool that is being increasingly used to diagnose and monitor several diseases and conditions in children $[13,14]$. The most commonly known use of PSG is diagnosing and monitoring sleep-disordered breathing (SDB), since it is the gold-standard method $[15,16]$. During full level 1 overnight video-PSG, a number of physiological signals are recorded. This includes electroencephalography (EEG), electro-oculogram, submental and leg electromyogram, oronasal airflow, abdominal and chest wall movements, pulse oximetry, partial pressure of end-tidal or transcutaneous carbon dioxide, and a video-recording [13,14].

\subsection{Polysomnography in preterm infants}

Cardiorespiratory monitoring is a key feature of contemporary neonatal intensive care. It enables the clinician to provide the best care for even the most preterm infants of 24 weeks' gestational age. Heart rate variability and general movement characteristics may serve as predictor for 
Table 1

Definitions of breathing abnormalities (according AASM criteria [67]).

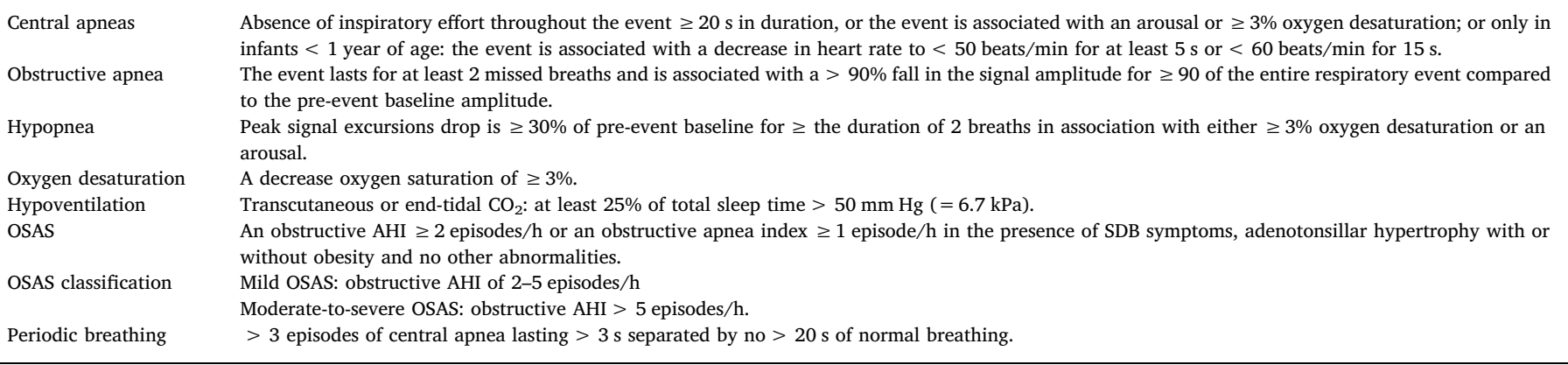

morbidity, mortality and neurodevelopmental outcome [17,18]. Nowadays, EEG and cardiorespiratory monitors can be used to evaluate sleep and sleep states in very small preterm infants $[19,20]$. PSG recording in the NICU is technically challenging, especially in smaller neonates. No international guidelines are available on timing of PSG in preterm infants. Very few studies have performed PSG in very low birthweight infants [21,22]. Performing full level 1 overnight video-PSG has some important limitations in preterm infants, since the stickiness of the recording electrodes can damage the fragile skin $[20,23]$ and the PSG equipment will give a lot of disturbance and interferes with the newborn individualized developmental care and assessment program (NIDCAP) guidelines for the sick preterm infant [24]. In a research setting, PSG can be performed in the neonatal intensive care unit (NICU) when an infant reaches the arbitrary age of 35 weeks' postmenstrual age (PMA) or when it is stable enough [25-28]. More feasible might be to perform a PSG near term equivalent age, commonly prior to discharge [8].

\section{Indications for polysomnography in preterm infants}

\subsection{Immature breathing pattern/persistent apnea of prematurity}

Apnea of prematurity is a common developmental disorder occurring in preterm infants [29]. It is a consequence of immaturity of the brainstem and peripheral chemoreceptors [30,31]. A preterm infant younger than 37 weeks' gestation is generally considered to be suffering of apnea of prematurity if it suffers apneas of $>20$ seconds' duration, or a shorter respiratory pause accompanied by bradycardia or hypoxia (Table 1 ). The incidence of apnea of prematurity is inversely related to gestational age. Virtually all preterm infants of a gestational age $<28$ weeks suffer from apnea of prematurity. In most infants, the symptoms resolve around 37 weeks' postmenstrual age (PMA).

Distinct from apnea of prematurity, periodic breathing is a normal immature breathing pattern for neonates and occurs in term as well as preterm infants. It appears in the second week after birth, peaks at several weeks of age, then decreases, but may continue for up to six months or longer [32]. Excessive periodic breathing ( $>10 \%$ of sleeping time) or an abrupt increase over prior baseline may reflect illness or physiological stressors, or may occur without any apparent clinical event. Nonetheless, its occurrence warrants consideration for potential pathology. Also, studies have reported increased cardiorespiratory events in preterm infants after immunizations, which might be related to periodic breathing. Others, however, have not found this association, which may reflect different maturational stages and clinical entities [33-35].

In many cases, occasional apneas or the possibility of an apnea may delay discharge from the hospital. The required apnea-free period needed before an infant can be discharged remains controversial. Clinicians typically assess maturation of respiratory control in relation with feeding behavior through observation of infants in the hospital with a duration varying between 1 and 21 days. In a retrospective study of 1403 infants with an average gestational age of $31.1 \pm 2.6$ weeks and an average PMA at discharge of $35.6 \pm 1.8$ week, the success rate and event identification rate for a specific apnea- or bradycardia-free interval was studied. Only those infants were studied who were ready-for-discharge and free from the last apnea or bradycardia event for 21 days; $84.2 \%$ did not have an apnea event and 78.5\% did not have a bradycardia event. For the entire cohort, a 95\% success rate was statistically reached, with a 7-day apnea- or bradycardia-free interval [5]. A higher success rate was found when the analysis was restricted to infants who were off methylxanthines for 3 or 5 days. Discontinuation of routine caffeine treatment was also studied in 95 preterm infants, and it was shown that after discontinuation a substantial number of hypoxic events persisted, but with increasing PMA these progressively decreased [36]. Extended caffeine treatment until 40 weeks' PMA decreased the number of hypoxic events.

Fairchild et al. provided a quantitative analysis of central apnea throughout the NICU stay of 1211 preterm infants born $<35$ weeks' gestation and concluded that NICU discharge was safe after a week free of needing stimulation to resolve an apneic spell, since most infants were being sent home without a monitor and very few, namely 4.7\% were readmitted within 2 months for an apnea or ALTE [37].

Some infants, however, continue to suffer from cardiorespiratory events. If apneas persist, other possible diagnoses must be excluded, for example central nervous system pathology. In rare cases, the congenital central hypoventilation syndrome (CCHS), or Ondine's curse, can be the cause of persisting apneas in infants [38]. The occurrence of apnea/bradycardia events after discharge from the hospital was studied in $>1000$ preterm and healthy term infants who were monitored at home. Extreme events, such as apnea $>30 \mathrm{~s}$ and/or heart rate $<60$ beats per minute for $>10 \mathrm{~s}$, were very rare but were observed most frequently in former preterm infants and the occurrence decreased until about 43 weeks' PMA [39].

In none of the studies described above, PSG was used to investigate the respiratory control in these preterm infants. Events were captured either through a nursing report in the medical chart [5], quantitative analysis of central apneas [40], or by home monitoring devices [39]. Only in a few studies polysomnography has been done in preterm infants before discharge from the hospital [8]. Daniëls et al. performed overnight polysomnography before discharge at a postmenstrual age of 36-40 weeks in 1274 preterm born infants with a gestational age < 34 weeks at birth and the findings were compared with data obtained subsequently from home documented monitoring [8]. It was found that 162 (12.7\%) infants still had central apneas $(>15 \mathrm{~s}$ ) and $34(2.7 \%)$ a bradycardia $(<50 \mathrm{bpm}$ for a minimum of $4 \mathrm{~s}$ ) and/or severe obstructive apnea for $>15 \mathrm{~s}$ with bradycardia $<60 \mathrm{bpm}$, with oxygen desaturation $<80 \%$. In follow-up, 19 out of 34 of these preterm infants had subsequent documented life threatening events caused by a central or obstructive apnea, with bradycardia varying from 12 to $41 \mathrm{bpm}$ during home monitoring, but none of the children with 
central apneas showed events at home. The conclusion of the authors was that identification of infants at risk with immature cardiorespiratory control, by means of polysomnography, and the follow up of infants at risk by memory cardiorespiratory home monitoring was proven to be a safe and a cost-effective strategy. The value of performing a polysomnography at discharge was confirmed in a study of 1058 infants in which 96 (9.1\%) infants needed cardiorespiratory home monitoring because of an abnormal polysomnography [41]. These infants were considered to be at high risk for developing acute events at home. Sixty-one infants showed alarms at home and 17 infants had serious alarms above the post-conceptional age of 50 weeks. The mean post-conceptional age when alarms stopped was 46 weeks. There was a significant negative correlation between the PMA at birth and the post-conceptional age at which the last alarm was noted.

Overall, preterm infants have been shown to have more apparent life threatening events compared to term infants. In a recent consensus of the American Academy of Pediatrics, a clinical practice guideline was provided regarding evaluation of the low-risk infant after experiencing a brief resolved unexplained event (BRUE), previously apparent life-threatening event (ALTE) [40]. This guideline only applies to low-risk infants experiencing a BRUE. The routine use of PSG in infants presenting with a lower-risk BRUE is not recommended. Infants born at a GA below 32 weeks are considered to be at high-risk and PSG may be considered in case of severe events.

In summary, is has been shown that a polysomnography before discharge from the hospital is useful in preterm infants to identify those infants who have abnormalities in their breathing pattern and who are at risk to get an event at home (Tables 1 and 3).

\section{Obstructive sleep apnea}

Preterm born infants are at a higher risk for obstructive sleep apnea [32,42,43]. Many apparently central apneas involve loss of airway tone that may result in intermittent airway obstruction and as a consequence prolong apnea. Infants with bronchopulmonary dysplasia (BPD) have more obstructive apneas observed in quiet sleep than infants without BPD [44]. Symptoms of obstructive sleep apnea may be subtle or absent in infants. These are snoring, noisy respirations, labored breathing, mouth breathing, and profuse sweating [45]. Occasionally, infants with obstructive sleep apnea may present with failure to thrive and developmental delay [46]. In childhood, preterm infants have a 3- to 5-fold elevated risk of developing obstructive sleep apnea syndrome (OSAS) compared to those without such history [47]. Evaluation by polysomnography showed a prevalence of $9.6 \%$ of obstructive sleep apnea syndrome among children born preterm at school age, while the prevalence in the control population was only $1 \%$ to $4 \%[42,48]$. A number of hypotheses on potential pathogenetic mechanisms may explain the higher prevalence of obstructive sleep apnea in preterm infants [47,49-52]. Facial asymmetry and elongated head shape can distort the dimensions and growth of the upper airway, resulting in a narrower upper airway [53,54]. In addition, prolonged use of an endotracheal tube and nasal feeding tube may change the shape of the palate and adversely affect upper airway growth. Generalized muscle hypotonia can also predispose preterm born infants to obstructive sleep apnea [49].

In summary, although a number of studies have been conducted to investigate the relationship between prematurity and obstructive sleep apnea, it is still difficult to determine whether a preterm infant is at risk for obstructive sleep apnea or not. If an infant shows clinical signs of obstructive sleep apnea or other related symptoms, such as failure to thrive, performing a PSG might be useful and care for the preterm born infant can be optimized.

\section{Bronchopulmonary dysplasia}

Bronchopulmonary dysplasia is a chronic lung disorder of preterm infants and the incidence of severe bronchopulmonary dysplasia has not decreased over the last decades because more immature infants survive the neonatal period due to improved perinatal care. In Europe, the incidence of BPD ranges from $4 \%$ in infants born at a gestational age of 31 weeks to $56 \%$ in infants born before 26 weeks' GA [55]. Children with BPD are at increased risk of long-term respiratory morbidity. Early assessment of both lung structure and ventilatory function may help to detect those children who are more likely to have severe respiratory problems and are at risk for serious respiratory morbidity in later life. Maintenance of appropriate blood oxygen saturation can improve growth, lower the risk of sudden death associated by hypoxia and may attenuate pulmonary hypertension [56-58]. In a study with a limited number of preterm infants with BPD, who were evaluated to rule out apnea or bradycardia prior to discharge from the hospital, significantly more central apneas and lower oxygen saturation levels were found compared to non-BPD preterms [28]. Interestingly, when supplemented with oxygen, BPD preterms had significantly higher saturation and both central apnea and periodic breathing densities declined significantly with this improvement in saturation. It was suggested that a better saturation status may improve central respiratory stability in chronic lung disease. However, no general consensus exists regarding the assessment of pulmonary reserve or the optimal timing for weaning home supplemental oxygen in infants with BPD. Oxygen saturations (SaO2) between 92\% and 96\% are considered desirable for optimal growth and development of preterm infants with BPD.

Polysomnography has been used as a proxy marker for ventilatory reserve and can be performed early in life, but studies are scarce [28,44,58-60]. McGrath-Morrow et al. studied a cohort of 62 stable preterm infants/children < 3 year (mean GA at birth 25 weeks) with chronic lung disease who were referred for overnight PSG [60]. The reason for PSG was assessment of supplemental oxygen needs and evaluation for the presence of sleep-disordered breathing. Fifty-five of the children (89\%) were on room air at the start of the study and 41 children remained on room air throughout the study, while 14 required the initiation of supplemental oxygen. Seven children were on supplemental oxygen at the start of the study and remained on supplemental oxygen. It was concluded that overnight PSG was more sensitive in assessing pulmonary reserve than outpatient clinical measures (respiratory rate and room air SaO2). This was also confirmed in a study of 63 infants with BPD; short-term (20-30 min), awake $\mathrm{SaO} 2$ measurements did not predict prolonged sleep SaO2 [58]. In this study, it was also shown that infants in whom $\mathrm{SaO} 2 \mathrm{remained}>92 \%$ a positive growth trend remained, compared to those with a SaO2 between 88 and $91 \%$.

In summary, brief pulse oximetry assessments during outpatient clinic visits may not be accurate enough to evaluate pulmonary reserve or assess supplemental oxygen needs [58,60-62] and overnight PSG registration is recommended before cessation of supplemental oxygen.

\section{Altered sleep}

Numerous studies have shown differences in sleep quality between preterm and full-term infants [46,63]. Preterm infants develop sleep-wake cycles differently from full-term infants, but maturation of sleep is related to both time since birth and gestational age [64]. Huang et al. studied the sleep of preterm infants at 6 months of post-natal age who were born between 24 and 36 weeks of gestational age [59]. Excluded were infants with bronchopulmonary dysplasia or infants who still required oxygen support (nasal cannula) after discharge. PSG data showed that $80.6 \%$ of the infants 
Table 2

Factors involved in the pathogenesis of the breathing pattern abnormalities in preterm infants.

\begin{tabular}{|c|c|c|}
\hline Central causes & Peripheral causes & Other causes \\
\hline Blunted response to a hypercapnic challenge & $\begin{array}{l}\text { Diminished glossopharyngeal muscular contraction in response to } \\
\text { periodic breathing }\end{array}$ & Chronic lung disease \\
\hline Hypoxic ventilatory depression & Poor sucking and swallowing that result in apnea & $\begin{array}{l}\text { Endotracheal tube and nasal feeding tube changing the } \\
\text { shape of the palate }\end{array}$ \\
\hline Decreased central chemosensitivity & Feeding related increase in apneas and desaturations & $\begin{array}{l}\text { Facial asymmetry and elongated head shape narrowing the } \\
\text { upper airway }\end{array}$ \\
\hline Delayed central nervous system development & $\begin{array}{l}\text { Lack of tone in the upper airway followed by collapse and } \\
\text { obstruction }\end{array}$ & \\
\hline
\end{tabular}

had an apnea-hypopnea index (AHI) $>1$ event/h (mean AHI = 3.63 \pm 3.24$)$, mean SaO2 $97.01 \pm 1.00 \%$, total sleep time $368.83 \pm 55.05$ min, sleep efficiency $82.43 \pm 14.66 \%$, and REM $24.03 \pm 6.43 \%$. Apart from this, preterm infants had longer nocturnal sleep duration at 6 months of age, more night awakenings, and longer daytime sleep duration, compared to full-term infants at 6 months of age. The caregivers of preterm infants noted that their children had more sleep problems. A significant difference between preterm and full-term infants in nocturnal sleep duration and loud-noisy breathing was noted.

\section{Conclusions}

There are a number of factors involved in the pathogenesis of breathing pattern abnormalities in preterm infants and the origin can be related to central, peripheral and other causes (Table 2). The total number of days with apnea and resolution of episodes at $>36$ weeks' postmenstrual age may have a worse neurodevelopmental outcome $[65,66]$. Apnea accompanied with both bradycardia and oxygen desaturation is common in nearly all preterm infants, despite respiratory and pharmacologic treatments administered. These apneas reflect immaturity of respiratory control and generally resolve by 36 to 37 weeks' postmenstrual age in infants born at $\geq 28$ weeks' gestation. Management of refractory apnea, optimal dosage and duration of various therapies, and determining readiness for discharge from the hospital are major challenges. A clinically significant apnea event-free period before discharge of 5 to 7 days is commonly used, although a longer period may be suitable for infants born at $<26$ weeks' gestation [29]. Preterm born infants may also suffer from obstructive sleep apneas which are sometimes difficult to recognize and can appear at later age. Accurate recognition and quantification of central and obstructive apnea using PSG may be helpful.

In children with bronchopulmonary dysplasia oxygen saturations between $92 \%$ and $96 \%$ are considered desirable for optimal growth and development of preterm infants with BPD. Brief pulse oximetry assessments during outpatient clinic visits may not be accurate enough to decide about discontinuation of oxygen therapy and PSG will give a better direction in the decision whether to stop or continue oxygen measurements. Finally, PSG measurements are helpful to determine the quality of sleep, which might be related to the development of the child. Table 3 gives an overview of the indications for PSG in preterm infants.

\section{Key guidelines}

- A PSG before discharge from the hospital is useful in preterm infants with persistent apneas and/or desaturations to identify those infants who have abnormalities in their breathing pattern and who are at risk to get an event at home.

- Preterm born infants are at risk for obstructive sleep apnea. If an infant shows clinical signs of obstructive sleep apnea or other related symptoms, such as failure to thrive, a PSG might be useful and care for the preterm born infant can be optimized.

- In infants with BPD, brief pulse oximetry assessments during outpatient clinic visits may not be accurate enough to evaluate pulmonary reserve or assess supplemental oxygen needs and overnight PSG registration is recommended before cessation of supplemental oxygen.

- PSG measurements are helpful to determine the quality of sleep, which might be related to the development of the child.

\section{Research directions}

Harmfulness of recurrent apnea, bradycardia, and hypoxemia in preterm infants on growth and neurocognitive outcome.

Significance of periodic breathing.

Timing of discontinuation of oxygen therapy in infants with chronic lung disease.

The value of actigraphy in relation to sleep status, sleep quality, growth and neurocognitive outcome.

Longitudinal PSG measurements in which sleep-breathing disorders or poor nocturnal sleep quality are related to neurocognitive development.

PSG breathing scoring criteria for the preterm infant.

Table 3

Indications for polysomnography.

$\begin{array}{ll}\text { Assessment of supplemental oxygen needs } & \begin{array}{l}\text { Cause of persisting apneas/ } \\ \text { bradycardia } \\ \text { Obstructive apnea detection } \\ \text { Seizure detection }\end{array} \\ \begin{array}{l}\text { Marker for ventilatory reserve } \\ \text { Cause of persisting pathological central } \\ \text { apnea }\end{array} & \begin{array}{l}\text { Sleep quality measurement } \\ \text { Gastro-esophageal reflux related } \\ \text { apnea }\end{array} \\ \begin{array}{l}\text { Determination of central hypoventilation } \\ \text { treatment }\end{array} & \end{array}$




\section{Conflict of interest}

\section{The authors report no conflict of interest.}

\section{References}

[1] B. Schmidt, R.S. Roberts, P. Davis, L.W. Doyle, K.J. Barrington, A. Ohlsson, et al., Caffeine therapy for apnea of prematurity, N. Engl. J. Med. 354 (20) (May 18 2006) $2112-2121$.

[2] B. Schmidt, R.S. Roberts, P. Davis, L.W. Doyle, K.J. Barrington, A. Ohlsson, et al., Long-term effects of caffeine therapy for apnea of prematurity, N. Engl. J. Med. 357 (19) (Nov 8 2007) 1893-1902.

[3] S.A. Prins, S.J. Pans, M.M. van Weissenbruch, F.J. Walther, S.H. Simons, Doxapram use for apnoea of prematurity in neonatal intensive care, Int. J. Pediatr. 2013 (2013) 251047.

[4] Y. Ogawa, M. Irikura, Y. Kobaru, M. Tomiyasu, Y. Kochiyama, M. Uriu, et al., Population pharmacokinetics of doxapram in low-birth-weight Japanese infants with apnea, Eur. J. Pediatr. 174 (4) (Apr 2015) 509-518.

[5] S.A. Lorch, L. Srinivasan, G.J. Escobar, Epidemiology of apnea and bradycardia resolution in premature infants, Pediatrics 128 (2) (Aug 2011) e366-e373.

[6] H. Daniels, H. Devlieger, T. Minami, E. Eggermont, P. Casaer, Infant feeding and cardiorespiratory maturation, Neuropediatrics 21 (1) (1990 Feb) 9-10.

[7] S. Sakonidou, J. Dhaliwal, The management of neonatal respiratory distress syndrome in preterm infants (European Consensus Guidelines—2013 update), Arch. Dis. Child. Educ. Pract. Ed. 100 (5) (Oct 2015) 257-259.

[8] H. Daniels, G. Naulaers, F. Deroost, H. Devlieger, Polysomnography and home documented monitoring of cardiorespiratory pattern, Arch. Dis. Child. 81 (5) (Nov 1999) 434-436.

[9] A.H. Jobe, E. Bancalari, Bronchopulmonary dysplasia, Am. J. Respir. Crit. Care Med. 163 (7) (2001) 1723-1729.

[10] E.C. Eichenwald, A. Aina, A.R. Stark, Apnea frequently persists beyond term gestation in infants delivered at 24 to 28 weeks, Pediatrics 100 (3 Pt 1) (Sep 1997) 354-359.

[11] R.S. Horne, G.M. Nixon, The role of physiological studies and apnoea monitoring in infants, Paediatr. Respir. Rev. 15 (4) (Dec 2014) $312-318$.

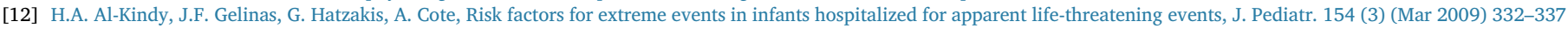
(7 e1-2).

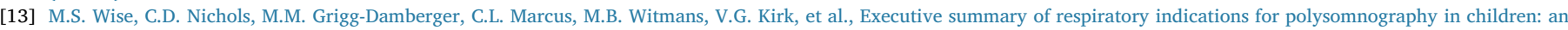
evidence-based review, Sleep 34 (3) (Mar 2011) 389-398AW.

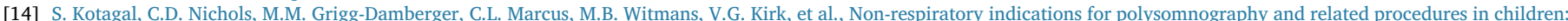
an evidence-based review, Sleep 35 (11) (Nov 01 2012) 1451-1466.

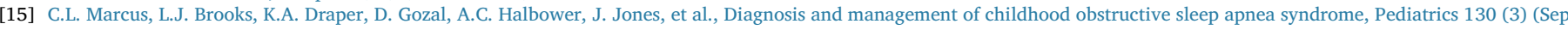
2012) 576-584 (Practice Guideline).

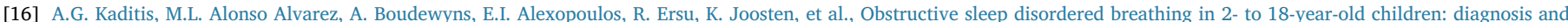
management, Eur. Respir. J. 47 (1) (Jan 2016) 69-94.

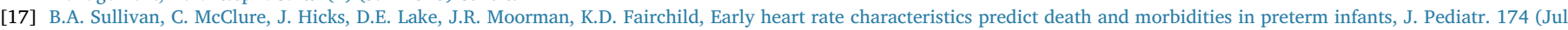
2016) 57-62.

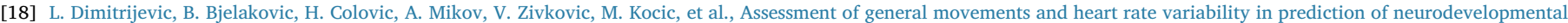
outcome in preterm infants, Early Hum. Dev. 99 (Aug 2016) 7-12.

[19] M.S. Scher, M.W. Johnson, D. Holditch-Davis, Cyclicity of neonatal sleep behaviors at 25 to 30 weeks' postconceptional age, Pediatr. Res. 57 (6) (Jun 2005) 879-882.

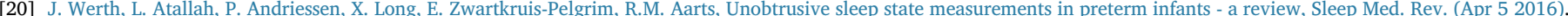

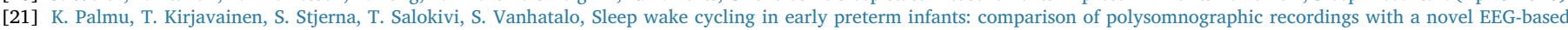
index, Clin. Neurophysiol. 124 (9) (Sep 2013) 1807-1814.

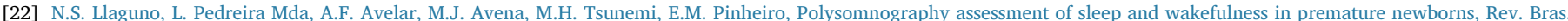
Enferm. 68 (6) (Nov-Dec 2015) 1109-1115.

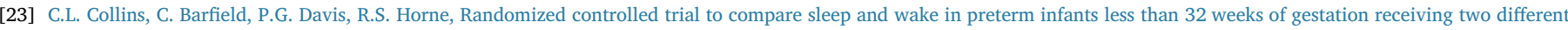
modes of non-invasive respiratory support, Early Hum. Dev. 91 (12) (Dec 2015) 701-704.

[24] K.A. Vandenberg, Individualized developmental care for high risk newborns in the NICU: a practice guideline, Early Hum. Dev. 83 (7) (Jul 2007) 433-442.

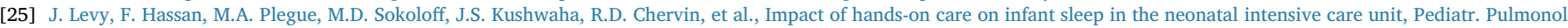
(Jun 30 2016).

[26] R.A. Shellhaas, J.W. Burns, J.D. Barks, R.D. Chervin, Quantitative sleep stage analyses as a window to neonatal neurologic function, Neurology 82 (5) (Feb 042014 ) $390-395$.

[27] A.J. Oliveira, M.L. Nunes, A. Fojo-Olmos, F.M. Reis, J.C. da Costa, Clinical correlates of periodic breathing in neonatal polysomnography, Clin. Neurophysiol. 115 (10) (Oct 2004) 2247-2251.

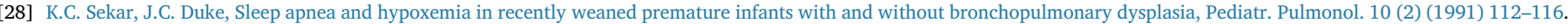

[29] E.C. Eichenwald, Committee on F, Newborn AAoP, Apnea of Prematurity, Pediatrics 137 (1) (Jan 2016).

[30] M. Katz-Salamon, Delayed chemoreceptor responses in infants with apnoea, Arch. Dis. Child. 89 (3) (Mar 2004) 261-266.

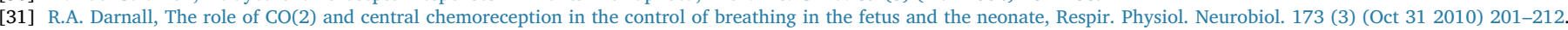

[32] P.B. Sharma, F. Baroody, D. Gozal, L.A. Lester, Obstructive sleep apnea in the formerly preterm infant: an overlooked diagnosis, Front. Neurol. 2 (2011) 73.

[33] A.K. Furck, J.W. Richter, E. Kattner, Very low birth weight infants have only few adverse events after timely immunization, J. Perinatol. 30 (2) (Feb 2010) 118-121.

[34] T. Carbone, B. McEntire, D. Kissin, D. Kelly, A. Steinschneider, K. Violaris, et al., Absence of an increase in cardiorespiratory events after diphtheria-tetanus-acellular pertussis immunization in preterm infants: a randomized, multicenter study, Pediatrics 121 (5) (May 2008) e1085-e1090.

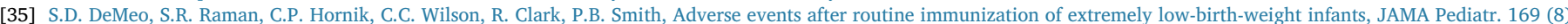
(Aug 2015) 740-745.

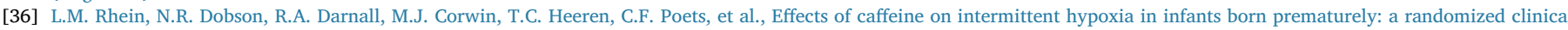
trial, JAMA Pediatr. 168 (3) (Mar 2014) 250-257.

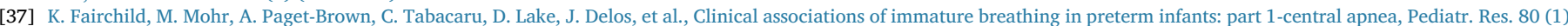
(2016 Jul) 21-27.

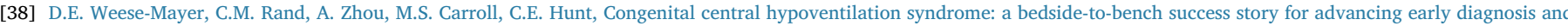
treatment and improved survival and quality of life, Pediatr. Res. 02 (Nov 2016).

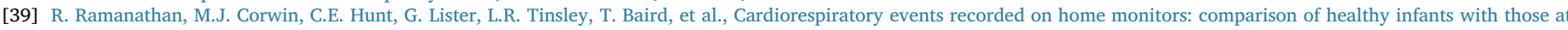
increased risk for SIDS, JAMA 285 (17) (May 2 2001) 2199-2207.

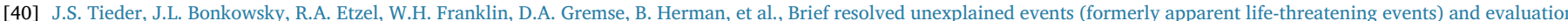
of lower-risk infants, Pediatrics 137 (5) (May 2016).

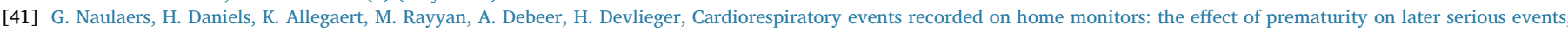
Acta Paediatr. 96 (2) (Feb 2007) 195-198.

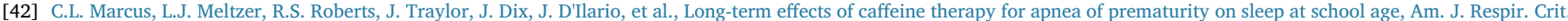
Care Med. 190 (7) (Oct 1 2014) 791-799.

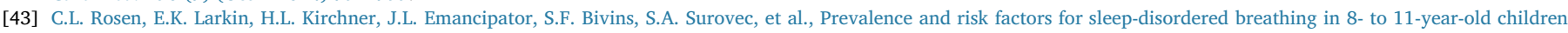
association with race and prematurity, J. Pediatr. 142 (4) (Apr 2003) 383-389.

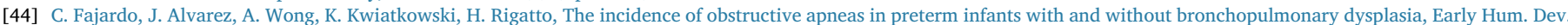
32 (2-3) (Mar 1993) 197-206.

[45] A. Kahn, J. Groswasser, M. Sottiaux, E. Rebuffat, P. Franco, Mechanisms of obstructive sleep apneas in infants, Biol. Neonate 65 (3-4) (1994) 235-239.

[46] A. Leiberman, A. Tal, I. Brama, S. Sofer, Obstructive sleep apnea in young infants, Int. J. Pediatr. Otorhinolaryngol. 16 (1) (Oct 1988) $39-44$.

[47] H.E. Montgomery-Downs, M.E. Young, M.A. Ross, M.J. Polak, S.K. Ritchie, S.K. Lynch, Sleep-disordered breathing symptoms frequency and growth among prematurely born infants, Sleep Med. 11 (3) (Mar 2010) 263-267 (Research Support, N.I.H., Extramural).

[48] J.C. Lumeng, R.D. Chervin, Epidemiology of pediatric obstructive sleep apnea, Proc. Am. Thorac. Soc. 5 (2) (Feb 15 2008) $242-252$.

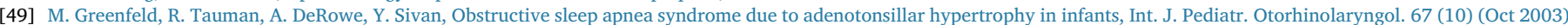
1055-1060.

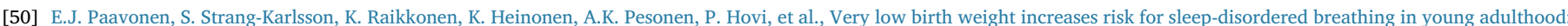


the Helsinki study of very low birth weight adults, Pediatrics 120 (4) (Oct 2007) 778-784.

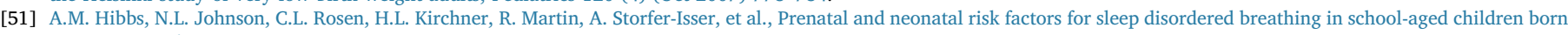
preterm, J. Pediatr. 153 (2) (Aug 2008) 176-182.

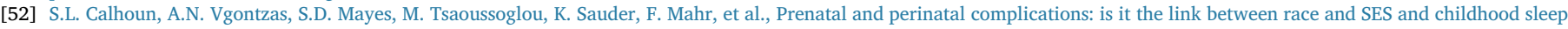
disordered breathing? J. Clin. Sleep Med. 6 (3) (Jun 15 2010) 264-269.

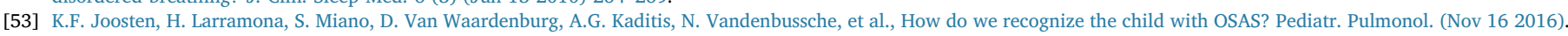

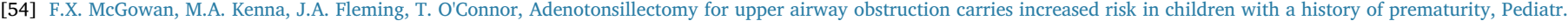
Pulmonol. 13 (4) (Aug 1992) 222-226.

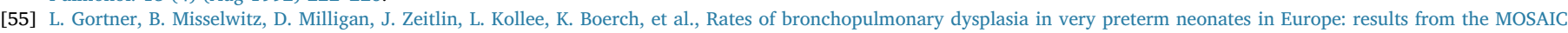
cohort, Neonatology 99 (2) (2011) 112-117 (Research Support, Non-U.S. Gov't).

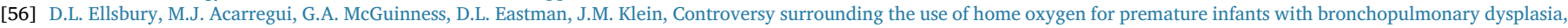
J. Perinatol. 24 (1) (Jan 2004) 36-40.

[57] P.J. Fleming, P.S. Blair, Sudden unexpected deaths after discharge from the neonatal intensive care unit, Semin. Neonatol. 8 (2) (Apr 2003) $159-167$.

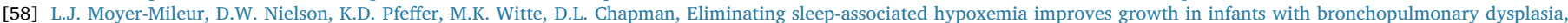
Pediatrics 98 (4 Pt 1) (Oct 1996) 779-783.

[59] Y.S. Huang, T. Paiva, J.F. Hsu, M.C. Kuo, C. Guilleminault, Sleep and breathing in premature infants at 6 months post-natal age, BMC Pediatr. 14 (Dec 162014$) 303$.

[60] S.A. McGrath-Morrow, T. Ryan, B.M. McGinley, S.O. Okelo, L.M. Sterni, J.M. Collaco, Polysomnography in preterm infants and children with chronic lung disease, Pediatr. Pulmonol. 47 (2) (Feb 2012) 172-179.

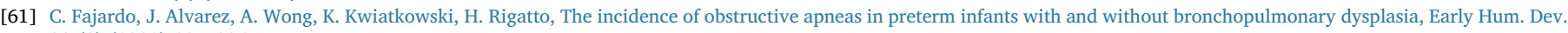
32 (2) (1993) 197-206.

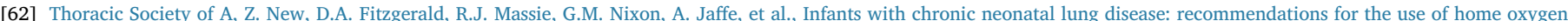
therapy, Med. J. Aust. 189 (10) (Nov 17 2008) 578-582.

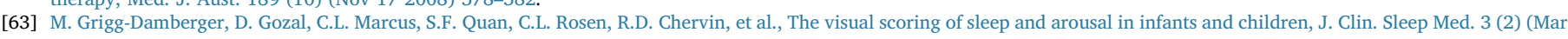
15 2007) 201-240.

[64] L. Curzi-Dascalova, P. Peirano, F. Morel-Kahn, Development of sleep states in normal premature and full-term newborns, Dev. Psychobiol. 21 (5) (1988 Jul) 431-444.

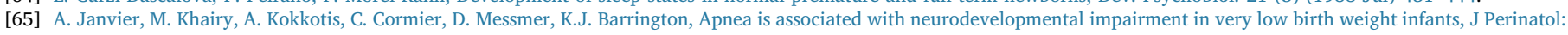
official journal of the California Perinatal Association 24 (12) (Dec 2004) 763-768.

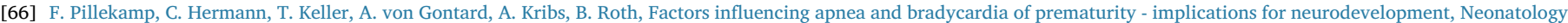
91 (3) (2007) 155-161.

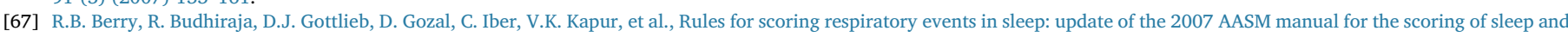
associated events. Deliberations of the Sleep Apnea Definitions Task Force of the American Academy of Sleep Medicine, J. Clin. Sleep Med. 8 (5) (Oct 15 2012) 597-619 (Practice Guideline).

Koen Joosten*

Erasmus MC, Sophia Children's Hospital, Pediatric Intensive Care, Rotterdam, The Netherlands E-mail address: k.joosten@erasmusmc.nl

Robbin de Goederen

Dutch Craniofacial Center, Department of Plastic, Reconstructive and Hand Surgery, Sophia Children's Hospital - Erasmus University Medical Center, Rotterdam, The Netherlands

Angelique Pijpers

Kempenhaeghe Academic Center for Epileptology, Sleep Medicine and Neurocognition, Heeze, The Netherlands

Karel Allegaert

Department of Pediatric Surgery, Erasmus University Medical Center - Sophia, Rotterdam, The Netherlands Department of Development and Regeneration, KU Leuven, Leuven, Belgium

* Corresponding author. 